International Journal of Applied Mathematics

Volume 33 No. $5 \quad 2020,929-936$

ISSN: 1311-1728 (printed version); ISSN: 1314-8060 (on-line version)

doi: http://dx.doi.org/10.12732/ijam.v33i5.13

\title{
MULTIPLICITY OF SOLUTIONS OF DIRICHLET'S PROBLEM FOR SECOND-ORDER $p$-LAPLACIAN DIFFERENTIAL EQUATIONS WITH VARIABLE COEFFICIENTS
}

\author{
Gergana Tcvetkova ${ }^{1}$, Stepan Tersian ${ }^{2}$ \\ ${ }^{1}$ Technical University of Varna \\ Dept. of Mathematics and Physics \\ Studentska Str. 1 \\ Varna - 9010, BULGARIA \\ ${ }^{2}$ Institute of Mathematics and Informatics \\ Bulgarian Academy of Sciences \\ Acad. G. Bonchev Str., Block 8 \\ Sofia - 1113, BULGARIA
}

\begin{abstract}
The existence of infinitely many solutions of Dirichlet's problem for $p$-Laplacian ordinary differential equation of second order is studied in the paper. The variational method is applied using the symmetric mountain pass theorem.
\end{abstract}

AMS Subject Classification: 34B15, 34B60, 49J35, 49J50

Key Words: $p$-Laplacian ODE's; symmetric mountain pass theorem; Sobolev space; weak solution

\section{Introduction}

In this paper we study the existence of solutions of the second-order $p$-Laplacian equation

$$
\left(\varphi_{p}\left(u^{\prime}(x)\right)\right)^{\prime}-a(x) \varphi_{q}(u(x))+b(x) \varphi_{r}(u(x))=0, \quad 0<x<L
$$

coupled with the Dirichlet conditions

Received: August 26, $2020 \quad$ (C) 2020 Academic Publications

$\S_{\text {Correspondence author }}$ 


$$
u(0)=u(L)=0 .
$$

We denote $\varphi_{p}(t)=|t|^{p-2} t, t \in \mathbb{R}$ for $p>1$ and assume that $1<p<q<r$, $a(x)$ and $b(x)$ are positive continuous functions on $[0, L]$. Partial cases of Eq. (1) are considered by many authors and appear in biomathematical and phase-transition models. Austin [2] considers the case $p=2, q=3, r=4$ in a model of an aneurysm in the circle of Willis. Grossinho and Sanchez [7] consider the periodic solutions of the equation in this case using variational method. Further periodic and homoclinic solutions are studied in [9]. Systems of $p$-Laplacian equations are considered in [4] using critical point theory for non-smooth functionals. Higher-order equations are studied in [8] using the generalized Clark's theorem. It is applied to fourth-order $p$-Laplacian equations in [10]. The case $p=2$ is considered in the thesis of Kalcheva [5], Chapter 2.

Further, by (P) will denote the problem (1) and (2). It has a variational structure, which means that its solutions can be obtained as critical points of a $C^{1}$ functional $J$ on a Sobolev space $X$, defined as

$$
X=W_{0}^{1, p}(0, L)=\left\{u \in L^{p}(0, L): u^{\prime} \in L^{p}(0, L), u(0)=u(L)=0\right\},
$$

where $L^{p}(0, L)$ is the usual Lebesque space. The space $X$ is a separable Banach space with norm

$$
\|u\|_{X}=\left(\int_{0}^{L}\left(\left|u^{\prime}(x)\right|^{p}+|u(x)|^{p}\right) \mathrm{d} x\right)^{\frac{1}{p}}
$$

which is equivalent to the norm

$$
\|u\|=\left(\int_{0}^{L}\left|u^{\prime}(x)\right|^{p} \mathrm{~d} x\right)^{\frac{1}{p}}
$$

by the Poincare inequality $\|u\|_{X} \leq C\left\|u^{\prime}\right\|_{L^{p}}$, where $\|u\|_{L^{p}}^{p}=\int_{0}^{L}|u|^{p} \mathrm{~d} x$.

Moreover, the embedding $X \in C([0, L])$ is compact, $u \in L^{q}(0, L)$ for $q \geq p$ and

$$
\int_{0}^{L}|u(x)|^{q} \mathrm{~d} x \leq\|u\|_{L^{\infty}}^{q-p}\|u\|_{L^{p}}^{p},
$$

(see [3], Chapter 8). We suppose that $a(x)$ and $b(x)$ are positive continuous functions and there are constants $a_{j}, b_{j}$ for $j=1,2$ such that

$$
0<a_{1} \leq a(x) \leq a_{2}, \quad 0<b_{1} \leq b(x) \leq b_{2} .
$$


The functional $J: X \rightarrow \mathbb{R}$ is defined as

$$
J(u)=\frac{1}{p} \int\left|u^{\prime}(x)\right|^{p} \mathrm{~d} x+\frac{1}{q} \int_{0}^{L} a(x)|u(x)|^{q} \mathrm{~d} x-\frac{1}{r} \int_{0}^{L} b(x)|u(x)|^{r} \mathrm{~d} x .
$$

Under conditions (5), $J$ is $C^{1}$ functional, which can be shown in a standard way (see [9],[5]) and

$$
\left\langle J^{\prime}(x), v\right\rangle=\int_{0}^{L}\left(\varphi_{p}\left(u^{\prime}\right) v^{\prime}+a(x) \varphi_{q}(u) v-b(x) \varphi_{r}(u) v\right) \mathrm{d} x .
$$

By a weak solution of problem (1) we mean a function $u \in X$, such that

$$
\int_{0}^{L}\left(\varphi_{p}\left(u^{\prime}\right) v^{\prime}+a(x) \varphi_{q}(u) v-b(x) \varphi_{r}(u) v\right) \mathrm{d} x=0
$$

i.e. $u$ is a critical point of $J$.

By a solution of problem $(\mathrm{P})$ we mean a function $u \in C([0, L])$, such that $\varphi_{p}\left(u^{\prime}\right) \in A C([0, L])$ and $u(x)$ satisfies Eq. (1) for $x \in[0, L]$ and boundary condition (2). Here $A C([0, L])$ denotes the space of absolutely continuous functions on $[0, L]$ (see $[1],[3])$. Let $u$ be a weak solution of $(\mathrm{P})$, i.e. let (6) hold. Since $w=\varphi_{p}\left(u^{\prime}\right) \in A C([0, L])=W^{1,1}(0, L)$,

$$
\int_{0}^{L} w v^{\prime} \mathrm{d} x=-\int_{0}^{L} w^{\prime} v \mathrm{~d} x
$$

for every $v \in X$. By $u^{\prime}=\varphi_{p^{\prime}}(w)=\varphi_{p}^{-1}(w) \in C([0, L])$, where $\frac{1}{p}+\frac{1}{p^{\prime}}=1$. It follows that

$$
\int_{0}^{L}\left(\left(\left|u^{\prime}\right|^{p-2} u^{\prime}\right)^{\prime}-a(x)|u|^{q-2} u+b(x)|u|^{r-2}|u|\right) v \mathrm{~d} x=0
$$

for every $v \in \mathbb{C}_{0}^{\infty}([0, L])$. Then it follows that $u$ is a solution of $(\mathrm{P})$. Further we look for critical points of functional $J$ in order to find the solution of $(\mathrm{P})$. We will apply variational method and symmetric mountain-pass theorem. Our main result is the following:

Theorem 1. Let $1<p<q<r, a(x)$ and $b(x)$ be positive continuous functions on $[0, L]$. Then the problem $(\mathrm{P})$ has infinitely many pairs of solutions.

The case $p>r$ is considered in [10] with application of the generalized Clark's theorem. The periodic and homoclinic solutions are studied in [9] in the case $p=2$ and for higher order equations in [8]. 
The paper is organized as follows. In Section 2 we present the variational formulation of the problem, formulate the symmetric mountain-pass theorem and prove a lemma for the $(P S)$ condition. In Section 3 we prove Theorem 1.

\section{Preliminary results}

As we mention in Introduction, we denote by $X$ the Sobolev space

$$
X=W_{0}^{1, p}(0, L)=\left\{u \in L^{p}(0, L): u^{\prime} \in L^{p}(0, L), u(0)=u(L)=0\right\}
$$

equipped with the norm

$$
\|u\|=\left(\int_{0}^{L}\left|u^{\prime}(x)\right|^{p} \mathrm{~d} x\right)^{\frac{1}{p}}
$$

We consider the functional $J: X \rightarrow \mathbb{R}$

$$
J(u)=\frac{1}{p} \int_{0}^{L}\left|u^{\prime}(x)\right|^{p} \mathrm{~d} x+\frac{1}{q} \int_{0}^{L} a(x)|u(x)|^{q} \mathrm{~d} x-\frac{1}{r} \int_{0}^{L} b(x)|u(x)|^{r} \mathrm{~d} x,
$$

and look for critical points of $J$, which are solutions of $(\mathrm{P})$. We will apply the following known result.

Theorem 2 ([11], Theorem 9.12). Let $E$ be an infinite dimensional Banach space and $I \in \mathbb{C}^{1}(E, \mathbb{R})$ be an even functional which satisfies Palais-Smale $(P S)$ condition, $I(0)=0$. If $E=V \bigoplus X$ where $V$ is finite dimensional and $I$ satisfies:

$\left(I_{1}\right)$ there are constants $\rho, \alpha>0$ such that $I(u) \geq \alpha$ if

$$
\|u\|_{E}=\rho, \quad u \in E
$$

( $\left.I_{2}\right)$ for each finite dimensional subspace $E_{n} \subset E$, there exists $R_{n}$, such that $I(u) \leq 0$ if $u \in E_{n},\|u\|_{E} \geq R_{n}$.

Then, I possesses an unbounded sequence of critical points.

Next, we formulate an inequality due to Lindqvist [6], used in [8] for the proof of $(P S)$ condition.

Lemma 1 ([6], Lemma 4.2). a) If $p \geq 2$ one has

$$
\left(\varphi_{p}(x)-\varphi_{p}(y)\right)(x-y) \geq \frac{2}{p\left(2^{p-1}-1\right)}|y-x|^{p}
$$

for $x, y \in \mathbb{R}$; 
b) If $1<p<2$

$$
\left(\varphi_{p}(x)-\varphi_{p}(y)\right)(x-y) \geq \frac{2}{p\left(2^{p-1}-1\right)}|y-x|^{p}
$$

where $C(p)$ depends only in $p$.

Now we prove the following auxiliary lemma.

Lemma 2. Let $1<p<q<r, a(x)$ and $b(x)$ be continuous positive functions on $[0, L]$ and (5) be satisfied. Then, the functional $J: X \rightarrow \mathbb{R}$ satisfies the $(P S)$ condition.

Proof. Let $\left\{u_{n}\right\}$ be a $(P S)$-sequence in $X$, i.e. $\left\{J\left(u_{n}\right)\right\}$ is a bounded sequence and $J^{\prime}\left(u_{n}\right) \rightarrow 0$ in $X^{\star}$. We have

$$
\frac{1}{r}\left\langle J^{\prime}\left(u_{n}\right), u_{n}\right\rangle=\frac{1}{r} \int_{0}^{L}\left(\left|u_{n}^{\prime}(x)\right|^{p}+a(x)\left|u_{n}(x)\right|^{q}-b(x)\left|u_{n}(x)\right|^{r}\right) \mathrm{d} x
$$

and

$$
\begin{aligned}
& J\left(u_{n}\right)-\frac{1}{r}\left\langle J^{\prime}\left(u_{n}\right), u_{n}\right\rangle \\
& =\left(\frac{1}{p}-\frac{1}{r}\right) \int_{0}^{L}\left|u_{n}^{\prime}(x)\right|^{p} \mathrm{~d} x+\left(\frac{1}{q}-\frac{1}{r}\right) \int_{0}^{L}\left|u_{n}(x)\right|^{q} \mathrm{~d} x \\
& \geq\left(\frac{1}{p}-\frac{1}{r}\right)\left\|u_{n}\right\|^{p},
\end{aligned}
$$

which implies that

$$
\left|J\left(u_{n}\right)\right|+\frac{1}{r}\left\|J^{\prime}\left(u_{n}\right)\right\|_{\star}\left\|u_{n}\right\| \geq\left(\frac{1}{p}-\frac{1}{r}\right) \mid u_{n} \|^{p} .
$$

Then, the sequence $\left\{u_{n}\right\}$ is bounded in $X$. Indeed, if we suppose that there is a subsequence $\left\{u_{n_{k}}\right\}$ still denoted by $\left\{u_{n}\right\}$ such that $\left\|u_{n}\right\| \rightarrow \infty$, by (7) we obtain

$$
\frac{\left|J\left(u_{n}\right)\right|}{\left\|u_{n}\right\|^{p}}+\frac{1}{r} \frac{\left\|J^{\prime}\left(u_{n}\right)\right\|_{\star}}{\left\|u_{n}\right\|^{p-1}} \geq \frac{1}{p}-\frac{1}{r}>0
$$

which implies a contradiction as $n \rightarrow \infty$, because $\left|J\left(u_{n}\right)\right|$ is bounded and $\left\|J^{\prime}\left(u_{n}\right)\right\|_{\star} \rightarrow 0$. Hence $\left\{u_{n}\right\}$ is a bounded sequence in $X$ and let $u_{n} \rightarrow u$ weakly in $X$. By compact embedding $X \subset C([0, L])$, it follows that:

$$
\lim _{n \rightarrow \infty} \int_{0}^{L} a(x)\left|u_{n}(x)\right|^{q} \mathrm{~d} x=\int_{0}^{L} a(x)|u(x)|^{q} \mathrm{~d} x
$$




$$
\lim _{n \rightarrow \infty} \int_{0}^{L} b(x)\left|u_{n}(x)\right|^{r} \mathrm{~d} x=\int_{0}^{L} b(x)|u(x)|^{r} \mathrm{~d} x .
$$

We have $\left\langle J^{\prime}\left(u_{n}\right), u_{n}\right\rangle \rightarrow 0$ as $n \rightarrow \infty$ by

$$
\left|\left\langle J^{\prime}\left(u_{n}\right), u_{n}\right\rangle\right| \leq\left\|J^{\prime}\left(u_{n}\right)\right\|_{\star}\left\|u_{n}\right\| \text {. }
$$

Then,

$$
\begin{aligned}
0=\lim _{n \rightarrow \infty}\left\langle J^{\prime}\left(u_{n}\right)-J^{\prime}(u), u_{n}-u\right\rangle & \\
& =\lim _{n \rightarrow \infty} \int_{0}^{L}\left(\varphi_{p}\left(u_{n}^{\prime}\right)-\varphi_{p}\left(u^{\prime}\right)\right)\left(u_{n}^{\prime}-u^{\prime}\right) \mathrm{d} x .
\end{aligned}
$$

By Lemma 1 , for $p \geq 2$ we have

$$
\left(\varphi_{p}\left(u_{n}^{\prime}\right)-\varphi_{p}\left(u^{\prime}\right)\right)\left(u_{n}^{\prime}-u^{\prime}\right) \geq \frac{2}{p\left(2^{p-2}-1\right)}\left|u_{n}^{\prime}-u^{\prime}\right|^{p}
$$

which implies by (9) that $u_{n} \rightarrow u$ strongly in $X$. If $1<p<2$ by Lemma 1 and Hölder's inequality we have

$$
\begin{aligned}
0=\lim _{n \rightarrow \infty} \int_{0}^{L}\left(\varphi_{p}\left(u_{n}^{\prime}\right)\right. & \left.-\varphi_{p}\left(u^{\prime}\right)\right)\left(u_{n}^{\prime}-u^{\prime}\right) \mathrm{d} x \\
& \geq \lim _{n \rightarrow \infty}\left(\left\|u_{n}^{\prime}\right\|_{\mathbb{L}^{p}}-\left\|u^{\prime}\right\|_{\mathbb{L}^{p}}\right)\left(\left\|u_{n}^{\prime}\right\|_{\mathbb{L}^{p}}^{p-1}-\left\|u^{\prime}\right\|_{\mathbb{L}^{p}}^{p-1}\right) \geq 0
\end{aligned}
$$

which shows that $\left\|u_{n}\right\| \rightarrow\|u\|$. Since $X$ is an uniformly confex Banach space (see [3]) and $u_{n} \rightarrow u$ weakly in $X$, then $u_{n} \rightarrow u$ strongly in $X$ which completes the proof of Lemma 2.

\section{Proof of the main result}

In this section we prove Theorem 1 and verify the geometric assumptions of Theorem 2.

Since $X=W_{0}^{1, p}(0, L) \subset C([0, L]) \subset L^{q}(0, L)$ for $q>1$ there are constants $k_{1}$ and $k_{2}$ such that for $u \in X$

$$
\begin{aligned}
& \|u\|_{L^{q}}=\left(\int_{0}^{L}|u(x)|^{q} \mathrm{~d} x\right)^{\frac{1}{q}} \leq k_{1}\|u\|, \\
& \|u\|_{L^{r}}=\left(\int_{0}^{L}|u(x)|^{r} \mathrm{~d} x\right)^{\frac{1}{r}} \leq k_{2}\|u\| .
\end{aligned}
$$




\section{Proof of Theorem 1}

We apply Theorem 2 with $V=\{0\}$. To show $\left(I_{1}\right)$ in $X$ we have by (5) and (10)

$$
\begin{array}{rl}
J(u)=\frac{1}{p}\|u\|^{p}+\frac{1}{q} \int_{0}^{L} a(x)|u|^{q} \mathrm{~d} x-\frac{1}{r} \int_{0}^{L} b(x)|u|^{r} & \mathrm{~d} x \\
& \geq \frac{1}{p}\|u\|^{p}-\frac{b_{2} k_{2}}{r}\|u\|^{r} .
\end{array}
$$

Since $r>p$, for $\|u\|=e$ sufficiently small there exists $\alpha>0$ such that $J(u) \geq \alpha>0$ if $\|u\|=\rho$.

To prove the condition $\left(I_{2}\right)$ of Theorem 2 , let $X_{n} \subset X$ be an $n$-dimensional subspace of $X$ and $v_{n} \in X_{n}$ is arbitrary point. Since $X_{n}$ is finite dimensional space there are constants $d_{j n}, j=1, \ldots, 4$ such that

$$
\begin{aligned}
& d_{1 n}\left\|v_{n}\right\| \leq\left\|v_{n}\right\|_{\mathbb{L}^{q}} \leq d_{2 n}\left\|v_{n}\right\|, \\
& d_{3 n}\left\|v_{n}\right\| \leq\left\|v_{n}\right\|_{\mathbb{L}^{r}} \leq d_{4 n}\left\|v_{n}\right\| .
\end{aligned}
$$

Then by (5) and last inequalities we have:

$$
\begin{aligned}
J\left(v_{n}\right)=\frac{1}{p}\left\|v_{n}\right\|^{p}+ & \frac{1}{q} \int_{0}^{L} a(x)\left|v_{n}(x)\right|^{q} \mathrm{~d} x-\frac{1}{r} \int_{0}^{L} b(x)\left|v_{n}(x)\right|^{r} \mathrm{~d} x \\
\leq & \frac{1}{p}\left\|v_{n}\right\|^{p}+\frac{a_{2} d_{2 n}^{q}}{q}\left\|v_{n}\right\|^{q}-\frac{b_{1} d_{3 n}^{r}}{r}\left\|v_{n}\right\|^{r} \\
& =\left\|v_{n}\right\|^{p}\left(\frac{1}{p}+\frac{a_{2} d_{2 n}^{q}}{q}\left\|v_{n}\right\|^{q-p}-\frac{b_{1} d_{3 n}^{r}}{r}\left\|v_{n}\right\|^{r-p}\right) .
\end{aligned}
$$

By $1<p<q<r$, there exists $R_{n}$ sufficiently large, such that $J\left(v_{n}\right) \leq 0$ for $\left\|v_{n}\right\| \geq R_{n}$. Then by Theorem 2 , the functional $J$ has infinitely many pairs of critical points.

\section{Acknowledgements}

The second author is supported by the Bulgarian National Science Fund under Project DN 12/4 'Advanced analytical and numerical methods for nonlinear differential equations with applications in finance and environmental pollution', (2017). His work is also in frames of the working program under the bilateral agreement between Bulgarian Academy of Sciences and Serbian Academy of Sciences and Arts (2020-2022). 


\section{References}

[1] R. Adams, Sobolev Spaces, Academic Press, New York (1975).

[2] G. Austin, Biomathematical model of aneurysm of the circle of Willis, I: The During equation and some approximate solutions, Math. Biosci., 11 (1971), 163-172.

[3] H. Brezis, Functional Analysis, Sobolev Spaces and Partial Differential Equations, Springer (2011).

[4] P. Jebelan, G. Morosanu, Mountain-pass type solutions for discontinuous peturbations of the vector $p$-Laplacian, Nonlinear Funct. Anal. Appl., 10 (2005), 591-611.

[5] E. Kalcheva, Periodic solutions of semilinear differential equations of second and fourth order, PhD Thesis, University of Ruse (2014).

[6] P. Lindqvist, On the equation $\operatorname{div}(|\nabla u|)^{p-2} \nabla u+\lambda|u|^{p-2} u=0$, Proc. Amer. Math. Soc., 109 (1990), 157-164.

[7] M. Grossinho, L. Sanchez, A note on periodic solutions of some nonautonomous differential equations, Bull. Aust. Math. Soc., 34 (1986), 253265.

[8] L. Saavedra, S. Tersian, Existence of solutions for 2nd-order nonlinear $p$ Laplacian differential equations, Nonlinear Analysis, Real World Appl., 34 (2017), 507-519.

[9] S. Tersian, J. Chaparova, Periodic and homoclinic solutions of extended Fisher-Kolmogorov equations, J. Math. Anal. Appl., 260 (2001), 490-506.

[10] S. Tersian, Existence of infinitely many solutions of problems for fourth order p-Laplacian differential equation, In: Proc. 48th Spring Conf. of Union of Bulg. Mathmaticians (2019), 27-34.

[11] P. Rabinowitz, Minimax methods in critical theory with applications to differential equations, In: CBMS Reg. Conf. Ser. Math. 65, AMS, Providence, Rhode Island (1986). 